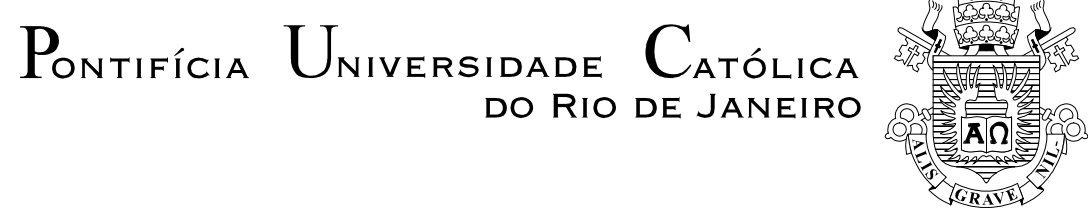

Victor Hugo Ayma Quirita

Estudo de Métodos Automáticos de Reconhecimento Facial para Vídeo Monitoramento

Dissertação de Mestrado

Dissertação apresentada como requisito parcial para obtenção do título de Mestre pelo Programa de PósGraduação em Engenharia Elétrica da PUC-Rio.

Orientador: Prof. Raul Queiroz Feitosa

Rio de Janeiro

Abril de 2014 


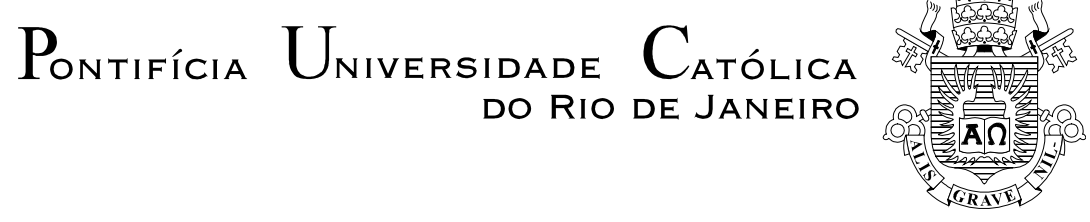

Victor Hugo Ayma Quirita

\title{
Estudo de Métodos Automáticos de Reconhecimento Facial para Vídeo Monitoramento
}

\begin{abstract}
Dissertação apresentada como requisito parcial para obtenção do título de Mestre pelo Programa de PósGraduação em Engenharia Elétrica da PUC-Rio. Aprovada pela Comissão Examinadora abaixo assinada.
\end{abstract}

Prof. Raul Queiroz Feitosa

Orientador

Departamento de Engenharia Elétrica - PUC-Rio

Dr. Gilson Alexandre Ostwald Pedro da Costa Departamento de Engenharia Elétrica - PUC-Rio

Prof. Bruno Feijo Departamento de Informática - PUC-Rio

Prof. José Eugenio Leal Coordenador Setorial do Centro Técnico Científico - PUC-Rio

Rio de Janeiro, 29 de abril de 2014 
Todos os direitos reservados. É proibida a reprodução total ou parcial do trabalho sem autorização da universidade, do autor e do orientador.

\section{Victor Hugo Ayma Quirita}

Nasceu em Cusco, Perú, no ano 1988. No 2011 obteve o seu diploma de graduação pela Universidade Nacional de San Antonio Abad del Cusco na especialidade de Engenharia Eletrônica. Atualmente esta fazendo 0 doutorado no programa de Engenharia Elétrica da PUCRio na área de Processamento de Sinais e Controle. Suas principais áreas de interesse são o processamento digital de imagens, visão computacional e biometria.

Ficha Catalográfica

Ayma Quirita, Victor Hugo

Estudo de Métodos Automáticos de Reconhecimento Facial para Vídeo Monitoramento / Victor Hugo Ayma Quirita ; orientador: Raul Queiroz Feitosa. - 2014.

77 f. : il. (color.) ; $30 \mathrm{~cm}$

Dissertação (mestrado)-Pontifícia Universidade Católica do Rio de Janeiro, Departamento de Engenharia Elétrica, 2014.

Incluí bibliografia.

1. Engenharia Elétrica - Teses. 2. Reconhecimento facial. 3. Vídeo monitoramento. 4. Biometria. I. Feitosa, Raul Queiroz. II. Pontifícia Universidade Católica do Rio de Janeiro. Departamento de Engenharia Elétrica. III. Título

CDD: 621.3 
A Deus, por me permitir desfrutar da sua glória infinita e ser a estrela que guia meu caminho.

A meus pais, Victor e Rina, por serem mestres de vida. Tudo o seu amor incondicional e confiança estão refletidos neste trabalho.

A meus irmãos, Andrés, Paola e Isa, pelo grande amor e apoio constantes. 


\section{Agradecimentos}

Ao meu orientador, o professor Raul Queiroz Feitosa, pela oportunidade, paciência, apoio, orientação e confiança no trabalho desenvolvido.

À PUC-Rio e aos seus professores do Departamento de Engenharia Elétrica.

À CAPES pelos auxílios concedidos.

Ao meu irmão Andrés, pelo apoio, por acreditar em mim e pelas palavras de confiança quando achava dificuldades no desenvolvimento desta pesquisa.

Aos meus amigos e colegas da PUC-Rio, pela amizade, apoio e alegrias.

Aos meus amigos em Cusco, pela sua grande amizade e palavras de encorajamento. 


\section{Resumo}

Ayma Quirita, Victor Hugo; Feitosa, Raul Queiroz. Estudo de Métodos Automáticos de Reconhecimento Facial para Vídeo Monitoramento. Rio de Janeiro, 2014. 77p. Dissertação de Mestrado - Departamento de Engenharia Elétrica, Pontifícia Universidade Católica do Rio de Janeiro.

Esta dissertação teve por objetivo comparar o desempenho de diversos algoritmos que representam o estado da arte em reconhecimento facial a imagens de sequências de vídeo. Três objetivos específicos foram perseguidos: desenvolver um método para determinar quando uma face está em posição frontal com respeito à câmera (detector de face frontal); avaliar a acurácia dos algoritmos de reconhecimento com base nas imagens faciais obtidas com ajuda do detector de face frontal; e, finalmente, identificar o algoritmo com melhor desempenho quando aplicado a tarefas de verificação e identificação. A comparação dos métodos de reconhecimento foi realizada adotando a seguinte metodologia: primeiro, foi criado um detector de face frontal que permitiu o captura das imagens faciais frontais; segundo, os algoritmos foram treinados e testados com a ajuda do facereclib, uma biblioteca desenvolvida pelo Grupo de Biometria no Instituto de Pesquisa IDIAP; terceiro, baseando-se nas curvas ROC e CMC como métricas, compararam-se os algoritmos de reconhecimento; e por ultimo, as análises dos resultados foram realizadas e as conclusões estão relatadas neste trabalho. Experimentos realizados sobre os bancos de vídeo: MOBIO, ChokePOINT, VidTIMIT, HONDA, e quatro fragmentos de diversos filmes, indicam que o Inter Session Variability Modeling e Gaussian Mixture Model são os algoritmos que fornecem a melhor acurácia quando são usados em tarefas tanto de verificação quanto de identificação, o que os indica como técnicas de reconhecimento viáveis para o vídeo monitoramento automático em vídeo.

\section{Palavras-chave}

Reconhecimento Facial; Biometria; Vídeo Monitoramento; Estimativa de Pose. 


\section{Abstract}

Ayma Quirita, Victor Hugo; Feitosa, Raul Queiroz (Advisor). An Evaluation of Automatic Face Recognition Methods for Surveillance. Rio de Janeiro, 2014. 77p. MSc. Dissertation - Departamento de Engenharia Elétrica, Pontifícia Universidade Católica do Rio de Janeiro.

This dissertation aimed to compare the performance of state-of-the-arte face recognition algorithms in facial images captured from multiple video sequences. Three specific objectives were pursued: to develop a method for determining when a face is in frontal position with respect to the camera (frontal face detector); to evaluate the accuracy for recognition algorithms based on the facial images obtained with the help of the frontal face detector; and finally, to identify the algorithm with better performance when applied to verification and identification tasks in video surveillance systems. The comparison of the recognition methods was performed adopting the following approach: first, a frontal face detector, which allowed the capture of facial images was created; second, the algorithms were trained and tested with the help of facereclib, a library developed by the Biometrics Group at the IDIAP Research Institute; third, $\mathrm{ROC}$ and $\mathrm{CMC}$ curves were used as metrics to compare the recognition algorithms; and finally, the results were analyzed and the conclusions were reported in this manuscript. Experiments conducted on the video datasets: MOBIO, ChokePOINT, VidTIMIT, HONDA, and four fragments of several films, indicate that the Inter-Session Variability Modelling and Gaussian Mixture Model algorithms provide the best accuracy on classification when the algorithms are used in verification and identification tasks, which indicates them as a good automatic recognition techniques for video surveillance applications.

\section{Keywords}

Face Recognition; Biometrics; Video Surveillance; Pose Estimation. 


\section{Sumário}

1 Introdução 13

1.1. Motivação 14

1.2. Objetivos 15

Objetivo Geral 15

$\begin{array}{ll}\text { Objetivos Específicos } & 16\end{array}$

1.3. Estrutura da Dissertação 16

2 Reconhecimento Facial $\quad 17$

2.1. Sistemas de Reconhecimento Facial 18

2.1.1. Localização de Face 18

2.1.2. Normalização 19

2.1.3. Extração de Atributos 19

2.1.4. Correspondência 20

2.2. Técnicas de Reconhecimento Facial 20

2.3. Desafios no Reconhecimento Facial Automático 21

2.4. Sistemas de Reconhecimento Facial: Aplicação no Vídeo Monitoramento 22

2.5. Contextualização do Trabalho 22

3 Fundamentos Teóricos 24

3.1. Autofaces 24

3.2. Fisherfaces 26

3.3. Bayesian Intrapersonal/Extrapersonal Classifier 28

3.4. Local Gabor Binary Pattern Histogram Sequence 30

3.5. Graph Matching 31

3.6. Gaussian Mixture Model 33

3.7. Inter Session-Variability Modeling 34

3.8. Probabilistic Linear Discriminant Analysis 35

4 Metodologia $\quad 37$

4.1. Bancos de Imagens Estáticas e Vídeo 37

4.2. Detector de Face Frontal 42

4.2.1. Detecção 43 
4.2.2. Extração de Atributos 45

$\begin{array}{ll}\text { 4.2.3. Determinação do Estado Frontal } & 47\end{array}$

4.3. Protótipo de Vídeo Monitoramento - LVC 53

4.4. Biblioteca FACERECLIB 55

4.5. Procedimento Experimental 57

4.5.1. Geração dos Bancos de Imagens Faciais Normalizadas 57

4.5.2. Definição de protocolos 59

4.5.3. Configuração dos Parâmetros dos Algoritmos de Reconhecimento 62

4.5.4. Análise dos Resultados e Elaboração das Conclusões 62

5 Resultados 66

5.1. Avaliação Baseada na Taxa de Igual Erro 66

5.2. Avaliação em Verificação $\quad 67$

5.3. Avaliação em Identificação 68

6 Conclusões $\quad 71$

6.1. Discussão $\quad 71$

6.2. Trabalhos Futuros $\quad 72$

$\begin{array}{ll}7 \text { Referências Bibliográficas } & 73\end{array}$ 


\section{Lista de figuras}

Figura 3. Normalização Geométrica e Fotométrica.

Figura 4. (a) Decomposição de RN no subespaço principal B e seu complemento ortogonal B, (b) espectro de autovalores típico e a sua divisão nos dois subespaços ortogonais.

Figura 5. Imagens de amostra do banco ORL.

Figura 6. Imagens de amostra do banco FEl.

Figura 7. Imagens de amostra do banco IMM.

Figura 8. Imagens de amostra do banco VidTIMIT. 40

Figura 9. Imagens de amostra do banco ChokePOINT. 41

Figura 10. Imagens de amostra do banco HONDA. 41

Figura 11. Imagens de mostra do banco MOBIO. 42

Figura 12. Etapas para a criação do detector de face frontal. 43

Figura 13. Localização dos pontos centrais dos olhos, nariz e boca. 44

Figura 14. (a) Imagem com face frontal, (b) Imagem com variação na arfagem. $\quad 45$

Figura 15. Triângulo e os ângulos gerados pela união dos olhos e boca 46

Figura 16. Variação da medida de similaridade S com respeito das variações em arfagem e guinada.

Figura 17. (a) Medidas de similaridade para o mesmo individuo com variações de arfagem e guinada, (b) Medidas de similaridade para diferentes indivíduos com posição frontal.

Figura 18. Medidas de similaridade versus atributos para o banco de imagens IMM. Em vermelho, mesmos indivíduos; em azul, indivíduos diferentes.

Figura 19. Medidas de similaridade versus os atributos para o banco de imagens FEl. Em vermelho, mesmos indivíduos; em azul, indivíduos diferentes.

Figura 20. Medidas de similaridade versus os atributos para o banco de imagens VidTIMIT. Em vermelho, mesmos indivíduos; em azul, indivíduos diferentes.

Figura 21. Curvas ROC para os atributos no banco de imagens IMM. 51

Figura 22. Curvas ROC para os atributos no banco de imagens FEl. 51

Figura 23. Curvas ROC para os atributos no banco de imagens VidTIMIT 52 
Figura 24. Imagens classificadas de frontais e não frontais. 53

Figura 26. Protótipo de Vídeo Monitoramento, interface gráfica. 55

Figura 27. Nas linhas, o número de indivíduos no banco de dados; e nas colunas, o número de imagens por individuo no banco de dados.

Figura 28. Da primeira a quarta linha, imagens faciais normalizadas correspondentes aos bancos HONDA, VidTIMIT, ChokePOINT e MOBIO. 58 Figura 29. Imagens faciais normalizadas correspondentes aos fragmentos de filmes.

Figura 30. Curvas ROC para os bancos faciais normalizados: (a) e (b) ORL, (c) e (d) FILMS, (d) e (e) FUSION.

Figura 31. Curvas CMC para os bancos faciais normalizados: (a) e (b) ORL, (c) e (d) FILMS, (d) e (e) FUSION. 


\section{Lista de tabelas}

Tabela 1. Parâmetros dos atributos usados para a construção das curvas ROC.

Tabela 2. Configuração 1 - Indivíduos por conjunto de Treinamento e Desenvolvimento.

60

Tabela 3. Configuração 1 - Imagens por individuo no Cadastro e Teste.

Tabela 4. Configuração 2 - Imagens por individuo no Treinamento, Cadastro e Teste.

Tabela 6. Resultados de avaliação das taxas de igual erro para os diferentes algoritmos nas duas configurações para os bancos de imagens ORL, FILMS e FUSION. 